\title{
Phylogenetic Analysis of Verticillium dahliae Vegetative Compatibility Groups
}

\author{
M. Collado-Romero, J. Mercado-Blanco, C. Olivares-García, and R. M. Jiménez-Díaz
}

First and second authors: Instituto de Agricultura Sostenible (IAS), Consejo Superior de Investigaciones Científicas (CSIC), Apartado 4084, 14080 Córdoba, Spain; third and fourth authors: IAS-CSIC and Escuela Técnica Superior de Ingenieros Agrónomos y Montes, Universidad de Córdoba, Edificio C-4 'Celestino Mutis’, Campus de Rabanales, Ctra. Madrid-Cádiz, km 396, 14071 Córdoba, Spain. Accepted for publication 3 June 2008.

\begin{abstract}
Collado-Romero, M., Mercado-Blanco, J., Olivares-García, C., and Jiménez-Díaz, R. M. 2008. Phylogenetic analysis of Verticillium dahliae vegetative compatibility groups. Phytopathology 98:1019-1028.

The evolutionary relationships among Verticillium dahliae vegetative compatibility (VCG) subgroups VCG1A, VCG1B, VCG2A, VCG2B, VCG4A, VCG4B, and VCG6 were investigated by parsimony analysis of amplified fragment length polymorphism (AFLP) fingerprints and sequences of six DNA regions (actin, $\beta$-tubulin, calmodulin, and histone 3 genes, the ITS 1 and 2 regions of the rDNA, and a $V$. dahliae-specific sequence), using 101 isolates of diverse host and geographic origin. Polymorphisms in gene sequences among isolates of different VCGs were very low and individual gene genealogies provided very little resolution at the VCG level. The combined analysis of all DNA regions differentiated all VCG subgroups except for isolates in VCG1A and VCG1B. VCG clonal lineages in $V$. dahliae and evolutionary relationships among
\end{abstract}

ABSTRACT

Knowledge of the genetic variation within and among pathogen populations, as well as the genetic relationships among these populations, is essential for development of disease management strategies (39). For populations of Verticillium dahliae Kleb., the causal agent of Verticillium wilts of hundreds of herbaceous and woody crops (44), studies during the last decade have demonstrated that genetic variation in the pathogen is higher than previously thought. Moreover, limited understanding of the nature of such variation and the genetic relationships among $V$. dahliae populations has hampered our understanding of Verticillium wilt development and the efficacy of their management $(4,33,47)$. In addition, severe Verticillium wilts have newly appeared on plants previously considered to be nonhosts of $V$. dahliae (i.e., cauliflower and lettuce) and studies demonstrated that isolates causing severe disease in one host are potentially derived from those infecting other hosts $(34,46,53)$.

Genetic variation in $V$. dahliae has been studied mainly by vegetative compatibility analysis and, to a lesser extent, analyses of fungal DNA. Vegetative compatibility refers to the het (heterokaryon incompatibility) loci-controlled ability of strains of a fungal species to undergo hyphal anastomosis and form stable heterokaryons. Fungal isolates that are vegetatively compatible are placed in the same vegetative compatibility group (VCG) $(33,38)$. Because $V$. dahliae is a haploid, asexually reproducing fungus, hyphal anastomosis is a prerequisite to genetic exchange and eventual parasexual genetic recombination among different

Corresponding author: R. M. Jiménez-Díaz; E-mail address: ag1jidir@uco.es

doi:10.1094/PHYTO-98-9-1019

(C) 2008 The American Phytopathological Society them were resolved independently by analyses of AFLP fingerprints, multiple gene genealogies, and the combined data set of AFLP fingerprinting and multiple gene genealogies. Two main lineages (I and II) were identified with lineage II comprising two closely related subgroups of VCGs. Lineage I included VCG1A, VCG1B, and VCG2B ${ }^{334}$; and lineage II included, VCG2A and VCG4B (subclade 1); and VCG2B ${ }^{824}$, VCG4A, and VCG6 (subclade 2). VCG subgroups were monophyletic except for VCG2B that appeared polyphyletic. Limiting the parsimony analysis either to AFLP fingerprints or DNA sequences would have obscured intraVCG differentiation. Therefore, the dual approach represented by the independent and combined analyses of AFLP fingerprints and DNA sequences was a highly valuable method for the identification of phylogenetic relationships at the intraspecific level in V. dahliae.

Additional keywords: defoliating pathotype, genetic diversity, Verticillium wilt.

isolates of the pathogen $(26,44)$. Consequently, $V$. dahliae isolates in different VCGs are often thought of as genetically isolated populations which may vary in a number of ecological, physiological, and virulence traits $(33,47)$.

Four main $V$. dahliae VCGs (VCG1, VCG2, VCG3, and VCG4) have been identified using complementing nitrate-nonutilizing (nit) mutants of isolates from diverse hosts and geographic origin worldwide $(5,13,30,33,52)$. Recently, a new VCG, VCG6, was identified among $V$. dahliae isolates infecting pepper in California (6). Based on the frequency and vigor of complementation, each of VCG1, VCG2, and VCG4 were further divided into two subgroups, designated A and B $(5,31,51)$. Moreover, isolates of VCG2B infecting artichoke in eastern-central Spain were differentiated further into two additional subgroups, designated VCG2Br if the isolates complemented with international reference VCG2B testers and $\mathrm{VCG} 2 \mathrm{Ba}$ if the complementation occurred with VCG2Br isolates but not with the reference testers (28).

Asexual reproduction in $V$. dahliae, which results in the whole genome being transmitted as a unit from one generation to the next, together with the low number of VCGs found so far in $V$. dahliae populations generally have been considered as an indication of clonal structure in this fungus. However, as for other asexually reproducing fungi such as Fusarium oxysporum, clonality of isolates within a VCG should be recognized with caution since the occurrence of an identical set of het loci may be coincidental, possibly arising by convergence rather than common descent $(1,2,38)$.

Phenetic analysis of amplified fragment length polymorphism (AFLP) fingerprinting using a large collection of $V$. dahliae isolates from different hosts demonstrated that isolates within a VCG subgroup (A and B) are genetically similar and AFLP 
clustering of isolates correlates with VCG subgroups (14). Based on molecular characterization, VCG subgroups were as distinct as were different VCGs. However, high molecular variability occurred within VCGs as indicated by phenetic analysis of AFLP fingerprinting and amplification of specific polymerase chain reaction (PCR) markers (14). For example, most VCG2B isolates produce an 824-bp marker previously associated with the cottonnondefoliating (ND) V. dahliae pathotype (designated VCG2B ${ }^{824}$ ) whereas other ND VCG2B isolates (designated VCG2B ${ }^{334}$ ) and isolates of VCG1B amplify a 334-bp marker previously associated with the cotton-defoliating (D) pathotype belonging to VCG1A $(14,15,41)$. Furthermore, ND VCG2B ${ }^{334}$ isolates were molecularly more similar to isolates in VCG1A than to those in VCG2B ${ }^{824}(14,28)$. Molecular variability among $V$. dahliae isolates within a VCG might derive from mutational processes, thus we would expect variants to result in a clonal lineage. Conversely, this variability might result from genetic recombination and those isolates would not be placed in the same clonal lineage (6). Also, the existence of bridging isolates able to form stable heterokaryons between isolates in different VCGs would eventually facilitate some gene flow between them (4). Therefore, the evolutionary potential within $V$. dahliae may be intimately related to VCGs $(24,39)$. This is relevant to disease control in terms of potential for emergence of new virulence or pathogenicity traits in natural populations of $V$. dahliae. However, whether or not genetic recombination occurs between $V$. dahliae isolates in different VCGs has not yet been addressed by phylogenetic analysis.

Phylogenetic analyses in fungal populations have been carried out using genealogies of highly conserved genes such as actin $(A c t), \beta$-tubulin $(\beta$-tub), calmodulin $(\mathrm{Cal})$, histone-3 (H3), and especially the internal transcribed sequences (ITS) of the nuclear ribosomal RNA gene cluster (rDNA) $(10,12,23,29)$. In particular, $\beta$-tub and the ITS have been used for phylogenetic analyses of Verticillium spp. $(8,19,43,46)$. On the other hand, analysis of AFLP fingerprints is an alternative approach to investigate molecular phylogenetics of closely related taxa $(20,25,45)$. Furthermore, reconstruction of evolutionary history in clonal fungal populations using gene genealogies can both identify patterns of descent in other types of molecular markers, such as whole genome AFLP analysis, and be combined with them to infer more finely resolved phylogenies $(3,9,22)$.

The main objective of this study was to determine the evolutionary relationships among the currently identified VCG subgroups of $V$. dahliae (i.e., VCG1A, VCG1B, VCG2A, VCG2B, VCG4A, VCG4B, and VCG6). A second objective was to test the hypothesis of monophyletic origin of each of those VCG subgroups. To that aim, we used a collection of isolates of diverse host source and geographic origin to carry out phylogenetic analyses of data from AFLP fingerprints or sequences of the six conserved DNA regions: Act, $\beta$-tub, $\mathrm{Cal}, \mathrm{H3}$, and ITS1 and 2 of the rDNA and a $V$. dahliae-specific DNA sequence (11); we also included analysis of the combined data.

\section{MATERIALS AND METHODS}

Verticillium spp. isolates, culture conditions, and DNA extraction. A collection of 101 isolates of $V$. dahliae and one of $V$. albo-atrum from diverse host and geographic origins were used in this study (Table 1). Isolates were stored on plum lactose yeast extract agar (PLYA) (54) covered with liquid paraffin at $4^{\circ} \mathrm{C}$ in the dark (7). Isolates are deposited in the culture collection of the Departamento de Protección de Cultivos, Instituto de Agricultura Sostenible, CSIC, Córdoba, Spain. Active cultures of isolates were obtained on water agar amended with chlorotetracycline $\left(0.3 \mathrm{~g} \mathrm{liter}^{-1}\right)$ and subsequent subculturing on potato dextrose agar (PDA) (250 $\mathrm{g}$ of unpeeled potato, $20 \mathrm{~g}$ of agar, and $20 \mathrm{~g}$ of glucose per liter of distilled water) or, alternatively, PLYA (54). DNA was extracted from mycelia obtained from 6- to 7-day-old cultures of Verticillium isolates in Czpeck-Dox broth (Difco Laboratories, Detroit, MI) or PLYA, according to ColladoRomero et al. (14). DNA purity and concentration were determined spectrophotometrically using a Biophotometer (Eppendorf AG, Hamburg, Germany) and by agarose gel electrophoresis according to standard procedures. DNA solutions were stored at $-20^{\circ} \mathrm{C}$ until used.

Vegetative compatibility grouping of isolates. Of the $101 \mathrm{~V}$. dahliae isolates, 69 were characterized to a VCG in previous studies $(14,15,28,36,37)$ and 28 were newly assigned to a VCG according to Korolev and Katan (35) and Collado-Romero et al. (14). Briefly, nitrate-nonutilizing (nit) mutants were generated on water agar chlorate and further tested on Czapek-Dox agar (CDA). nit mutants were phenotyped on CDA amended with sodium nitrite $\left(0.5 \mathrm{~g} \mathrm{liter}^{-1}\right)$ or hypoxanthine $\left(0.2 \mathrm{~g} \mathrm{liter}^{-1}\right)(17)$. Then, nit 1 and NitM mutants from each isolate were used to test for heterokaryon self-compatibility as well as for complementation with nit mutants of the international Ohio Agricultural Research and Development Center (OARDC, The Ohio State University, Wooster, $\mathrm{OH}$ ) reference strains of $V$. dahliae VCGs (VCG1, VCG3, and VCG4A) and Israeli nit testers (VCG1A, VCG2A, VCG2B, and VCG4B) (36) in all possible combinations. nit mutants derived from reference strain $\mathrm{T} 9$ of $\mathrm{D}$ pathotype, kindly provided by T. Katan (The Volcani Center, Bet Dagan, Israel), were used to identify VCG1A. Since T9 belongs to VCG1A of Bell (1994), we assumed that isolates strongly compatible with it should be assigned to VCG1A. No complementation tests were done in this work to type isolates to VCG1B. VCG1B isolates provided and used in the study were tested for virulence to cotton and proved of the ND pathotype (R. M. Jiménez-Díaz, unpublished data). Israeli nit testers that were previously demonstrated to correlate with the international OARDC reference strains of $V$. dahliae VCGs were used for identification of VCG2A, VCG2B, and VCG4B $(33,36,47)$. Cultures were incubated at $25^{\circ} \mathrm{C}$ and a 12 -h photoperiod of fluorescent and near-UV light at $36 \mu \mathrm{Em}^{-2}$ for up to 4 weeks. Unknown isolates were assigned to a VCG when a dense, aerial growth or black microsclerotia formed where mycelia from the unknowns and the VCG tester strain had met.

AFLP assays, scoring of AFLP fragments and binary matrix generation. Eighty-nine $V$. dahliae isolates representative of $V$. dahliae VCG1A, VCG1B, VCG2A, VCG2B, VCG4A, VCG4B, and VCG6, and the single isolate of V. albo-atrum were selected for phylogenetic analysis using AFLP markers (Table 1). AFLP profiles of 40 Verticillium isolates (39 V. dahliae and 1 V. albo-atrum) were available from a previous study (14). Profiles of the remaining $50 \mathrm{~V}$. dahliae isolates were obtained in this study.

AFLP assays were performed using a fluorescent procedure as described in Collado-Romero et al. (14). Briefly, DNA was digested with $M s e I$ and EcoRI endonucleases and the restricted DNA fragments were ligated to $M s e I$ and EcoRI adaptors using T4 DNA ligase. A 5\% dilution of the ligation reaction in $\mathrm{TE}_{0.1}$ buffer was used for preselective amplification using $0.5 \mu \mathrm{l}$ of preselective primers $M s e \mathrm{I}-\mathrm{C}$ and EcoRI-0 and $15 \mu \mathrm{l}$ of the AFLP Core Mix included in the kit. Selective PCR amplifications were done using four EcoRI-XX-Dye/MseI-C combinations in independent reactions using fluorescently labeled dyes NED, JOE, and FAM: EcoRI-AT-NED, EcoRI-AG-JOE, EcoRI-GC-FAM, and EcoRI-GA-FAM. Artifacts in the AFLP profiles were avoided by replicating the assays twice for arbitrarily selected $V$. dahliae isolates V562I and 1F20I. Products of selective amplifications were separated by capillary electrophoresis in an automatic DNA sequencer (ABI Prism 3100 Genetic Analyzer, Applied Biosystems). The size of products was determined using GeneScan500 [ROX] (Applied Biosystems, Foster City, CA) as internal size standard (35 to $500 \mathrm{bp}$ ). One AFLP profile for each of the four selective primer combinations used, ranging from 50 to $490 \mathrm{bp}$, 
were established for each of isolates in the study using the GeneScan v. 2.0 analysis software (Applied Biosystems).

AFLP profiles were assessed individually for each isolate. Only unambiguous peaks were scored for presence or absence $(1=$ presence or $0=$ absence) using Genotyper software version 2.5 (Applied Biosystems). All DNA fragments within the range of 50 to 490 bp were selected first using the "unmark overlapping peaks' option and AFLP profiles were rescaled using the 'normalized scale option'. Unambiguous, consistent peaks, which scaled higher than 100 fluorescent units, were then selected and those inconsistent were deleted manually. A binary character matrix was developed by combining all data and used for subsequent phylogenetic analysis. Thereafter, data of AFLP and DNA sequence where used jointly for phylogenetic analysis (see below).

Phylogenetic analysis of AFLP patterns. Phylogenetic analysis of the AFLP patterns were conducted by maximum parsimony (MP) using phylogenetic analysis using parsimony (PAUP*) version 4.0beta10 (Sinauer Associates, Inc., Publishers, Sunderland, MA). Unweighted MP analysis was conducted with the heuristic search option and 10 random addition sequences with the MulTrees option on and TBR branch swapping. A strict consensus tree was inferred from most all parsimonious trees. Bootstrap support was calculated with 1,000 bootstrap replicates, using the same settings as for the general heuristic search analysis. The tree derived from MP analysis was rooted to $V$. albo-atrum.

Specific DNA sequences amplification and sequencing. Fifty $V$. dahliae isolates representing diversity in AFLP profiles among and within VCGs were selected for phylogenetic analysis using the polymorphic $V$. dahliae-specific sequence of $523 / 539 \mathrm{bp}$ $(11,15,40)$ (Table 1). Then, 17 of the 50 isolates representative of all molecular groups and VCGs and the single V. albo-atrum isolate were arbitrarily selected for phylogenetic analysis using sequences of genes actin (Act), calmodulin $(\mathrm{Cal}), \beta$-tubulin $(\beta$ $t u b)$, and histone $3(H 3)$, as well as the ITS 1 and 2 regions of the rDNA and the $V$. dahliae-specific polymorphic DNA sequence. All sequences were considered independent loci. Primers used for DNA amplification and sequencing were: Act, ACT-512F (ATGTGCAAGGCCGGTTTCGC) and ACT-783R (TACGAGTCCTTCTGGCCCAT) (10); $\beta$-tub, Bt1a (TTCCCCCGTCTCCACTTCTTCATG) and Bt1b (GACGAGATCGTTCATGTTGAACTC) (23); Cal, CAL-228F (GAGTTCAAGGAGGCCTTCTCCC) and CAL-737R (CATCTTTCTGGCCATCATGG) (10); H3, H3-1a (ACTAAGCAGACCGCCCGCAGG) and H3-1b (GCGGGCGAGCTGGATGTCCTT) (23); and ITS1 and 2 regions, Tavo1 (CTCATAACCCTTTGTGAACC) and Tavo2 (CCGAGGTCAACCGTTGCCG) (56). The translation elongation factor $1 \alpha(E F 1 \alpha)$ gene was also tested but amplification was unsuccessful (EF1: TGGGTAAGGAA/GGACAAGAC and EF2: GGAA/GGTACCAGTC/GATCATGTT) (42). Primers DB19 (CGGTGACATAATACTGAGAG) and DB22 (GACGATGCGGATTGAACGAA) (11) were used for the amplification and sequencing of the $V$. dahliae-specific sequence.

For all primer pairs, the reaction mixture $(25 \mu \mathrm{l})$ consisted of: $20 \mathrm{ng}$ of fungal DNA, $400 \mathrm{nM}$ of each primer, $200 \mathrm{nM}$ of each dNTP, $2 \mathrm{mM} \mathrm{MgCl} 2,2.5 \mu \mathrm{l} 10 \times$ reaction buffer and $0.75 \mathrm{U}$ DNA polymerase (Biotools, B\&M Labs, Madrid, Spain). The PCR cycling protocols were: denaturation at $94^{\circ} \mathrm{C}$ for $5 \mathrm{~min}$ followed by 35 cycles of $1 \mathrm{~min}$ at $94^{\circ} \mathrm{C}, 1 \mathrm{~min}$ at $55^{\circ} \mathrm{C}$ (for primer pairs ACT-512F/ACT783R, Bt1a/Bt1b, CAL228F/CAL737R, and $\mathrm{DB} 19 / \mathrm{DB} 22$ ) or $58^{\circ} \mathrm{C}$ (for primers pairs $\mathrm{H} 3-1 \mathrm{a} / \mathrm{H} 3-1 \mathrm{~b}$ and Tavo1/Tavo2), $1 \mathrm{~min}$ at $72^{\circ} \mathrm{C}$, and a final step of $6 \mathrm{~min}$ at $72^{\circ} \mathrm{C}$. Amplification with primer pair EF1/EF2 was tested at an annealing temperature range of 52 to $64^{\circ} \mathrm{C}$. The amplification products $(5 \mu \mathrm{l})$ were separated by electrophoresis on $1 \%$ agarose gels in $1 \times$ TAE buffer, stained with ethidium bromide and visualized under UV light. The 0.1-kb DNA ladder XIV size marker was used for electrophoresis (Roche Diagnostics, Mannheim, Germany). If only one band was identified in agarose gels, the amplicon was excised from the gel and purified using the QIAquick PCR purification kit (Qiagen $\mathrm{GmbH}$, Hilden, Germany). Amplification products were sequenced at Servicio de Secuenciación de ADN, Centro de Investigaciones Biológicas, Consejo Superior de Investigaciones Científicas, Madrid, Spain. Forward primers were used in all cases and both forward and reverse sequencing was done occasionally. Sequences were edited with EditSeq (Lasergene, Madison, WI). A search for sequence similarities was performed with the BLAST version 2.2.10 program of the National Center for Biotechnology Information (NCBI) network service.

DNA sequence analysis. All assayed loci except EFl $\alpha$ produced amplicons. Sequences of each of the loci were aligned using the CLUSTALW implemented in the MegAlign software (Lasergene, Madison, WI). For comparative purposes, two phylogenetic analyses were performed independently with loci Act, $\beta$-tub, Cal, H3, and ITS, as well as the V. dahliae-specific DNA sequence. First, data were analyzed by neighbor joining (NJ) (49) using TREECON version $1.3 \mathrm{~b}$ software and genetic distances (32) with indels being considered as changes in the sequence (55). Then, MP analysis was done with PAUP* version 4.0beta10 software using the same parameters as for AFLP analysis but increasing to 1,000 the number of random repeats in the heuristic search and bootstrap replicates. All trees, except those derived from the $V$. dahliae-specific sequence, were rooted using the $V$. albo-atrum sequences. Trees derived from analyses using the $V$. dahliae-specific sequence were rooted with the sequence of $V$. dahliae isolate V138I.

Trees for each of the loci were evaluated simultaneously for congruence using the partition homogeneity test (PHT) $(21,27)$ implemented in PAUP* version 4.0beta10. Act, $\beta$-tub, Cal, H3, and ITS sequences were combined to perform MP analysis using PAUP* version 4.0 beta10 and the same parameters considered in the analysis of each locus. For the combined analysis, indels within each loci were considered numeric characters (i.e., 0, 1, and 2): 0 , deletion; 1 , presence of nucleotide or nucleotides (insertion); and 2, presence of polymorphism in the insertion. Similarly, the combined sequences of loci Act, $\beta$-tub, Cal, H3, and ITS, and the $V$. dahliae-specific sequence were used to perform MP analysis.

Phylogenetic analysis of the combined data set of AFLP fingerprints and DNA sequences. Congruence of phylogenetic signals between AFLP data and DNA sequences was studied performing the PHT implemented in PAUP* version 4.0beta10. The combined set of AFLP and DNA sequence data from $V$. dahliae isolates was used for phylogenetic MP analysis using PAUP* version 4.0 beta10 with bootstrap analysis of 500 replicates. Parameters used in that analysis were the same as those used in the analysis of each locus. Indels within loci were coded as numerical characters $(0,1$, and 2$)$.

\section{RESULTS}

Vegetative compatibility grouping of isolates. Of the $28 \mathrm{~V}$. dahliae isolates newly characterized to VCG, 10 were assigned to VCG1A, 9 to VCG2A, 3 to VCG2B ${ }^{824}$, and 6 to VCG4B (Table 1). Six of the $10 \mathrm{VCG} 1 \mathrm{~A}$ isolates originated from cotton in Greece and four were from olive in Spain. VCG2A isolates were of diverse host and geographic origin, including one from each of cotton soil, melon, olive, potato, pumpkin, and watermelon in Spain; one from potato in Israel, one from broccoli in Germany, and one from Japan. The host of the isolate from Japan was unknown. The three isolates of VCG2B originated from chickpea in Italy, or strawberry in England. Finally, of six isolates assigned to VCG4B, one originated from tomato in Brazil, one from olive in Greece, one each from potato and eggplant from Israel, and two from strawberry in England. 
Polymorphisms in the $V$. dahliae-specific sequence. An isolate of $V$. albo-atrum did not yield any PCR product using primer pair DB19/DB22. Sequencing of the 523/539-bp amplicons from the 50 selected $V$. dahliae isolates produced three of the

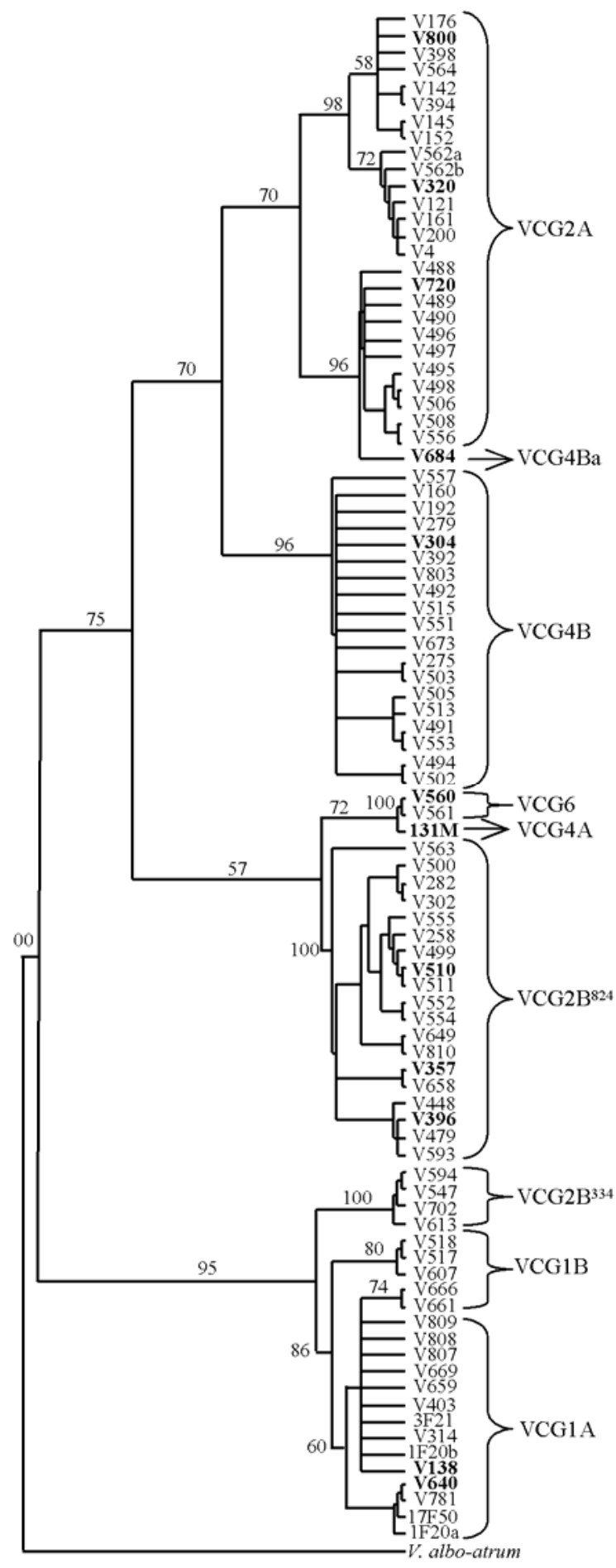

Fig. 1. Consensus tree inferred from maximum parsimony analysis using PAUP version 4.0 beta10 of 578 amplified fragment length polymorphism markers from 89 Verticillium dahliae isolates representative of different vegetative compatibility groups (VCG), V. albo-atrum was used to root the trees. Bootstraps values (1,000 replicates) are indicated in nodes. Parameters defining the tree are: tree length $(\mathrm{TL})=1,263$ steps, consistency index $(\mathrm{CI})=$ 0.4537 , retention index $(\mathrm{RI})=0.8129$, and rescaled consistency index $(\mathrm{RC})=$ 0.3688. VCG4Ba, artichoke isolate of VCG4B; VCG2B ${ }^{334}$, artichoke isolates of VCG2Br and VCG2Ba that amplified a 334-bp polymerase chain reaction (PCR) marker $(28 ; 40)$; VCG2B ${ }^{824}$, isolates of VCG2B that amplified a 824-bp PCR marker $(14,41)$. In bold, isolates that were used for phylogenetic analysis using DNA sequences. five polymorphic sequences described by Collins et al. (15), i.e., seq1, seq2, and seq4, as well as a new polymorphic sequence, namely seq 7 (Table 1). The complete sequences were deposited in the GenBank nucleotide database with accession numbers: seq1 (527 bp), DQ2666246; seq2 (526 bp), DQ2666247; DQ2666248; seq4 (542 bp), DQ2666249 and DQ2666244; and seq7 (541 bp), DQ2666245. The seq sequences and VCG of isolates were correlated as follows: seql was found only in isolates of VCG2B ${ }^{824}$ and VCG6, seq2 was amplified from isolates of VCG2A and VCG4B; and seq4 occurred in isolates of VCG1A, VCG1B, and VCG $2 B^{334}$. The new sequence seq 7 was only amplified from the two VCG4A isolates used in the study (Table 1).

Phylogenetic analysis of the four seq sequences yielded one most parsimonious tree only (not shown). In that tree, seql and seq 2 were the most closely related, seq 7 was intermediate and seq4 was most distant. This suggests that VCG2B ${ }^{824}$ and VCG6 are closely related to VCG2A and VCG4B, whereas VCG1A, VCG1B, and VCG2 $\mathrm{B}^{334}$ appear to be related to each other but represent a different, more distant clade, and VCG4A occupying an intermediate position between these two larger groups.

Phylogenetic analysis of the AFLP patterns. AFLP analysis of the complete set including $89 \mathrm{~V}$. dahliae isolates and one $\mathrm{V}$. albo-atrum in this study yielded 578 unambiguous and easily discernible AFLP fragments, of which 375 were informative for the MP analysis. Only 20,000 of a larger number of most parsimonious trees were retained to generate a consensus tree (Fig. 1). Clades resolved by MP analysis correlated with VCGs and were supported by bootstrap values higher than $70 \%$, except for the clade comprising isolates of VCG2B ${ }^{824}$, VCG6, and VCG4A (bootstrap 57\%). VCG1A and VCG1B were not clearly resolved but the VCG1A/1B group was closely related to the VCG2B ${ }^{334}$ clade. Isolates of VCG2A were separated into two clades, each of them with high bootstrap support. There was no correlation of the two VCG2A clades with host or geographic origin of isolates. VCG4B isolates comprised a main clade closely related to the VCG2A clades, except for the VCG4B isolate, V684I, from artichoke, which was actually placed in one of VCG2A clades. Isolates of VCG2B ${ }^{824}$ were placed in a clade closely related to that comprising isolates of VCG4A and VCG6. It was noteworthy that isolates of VCG2 subgroups A and B were less related to each other than they were to other VCG subgroups (i.e., VCG4B or VCG4A and VCG6), and same occurred with isolates of VCG4 subgroups.

Characterization of amplified gene sequences. PCR assays using DNA from Verticillium spp. isolates produced a single amplification product for each of genes Act, $\beta$-tub, $\mathrm{Cal}$, and $\mathrm{H} 3$, and the ITS regions of rDNA. Gene identity of the amplified sequences was confirmed by comparison with the GenBank databases. None of the Verticillium spp. isolates yielded any PCR product using primer pair EF1/EF2. Amplifications from $V$. alboatrum isolate V048I from hops yielded DNA fragments of $200 \mathrm{bp}$ (GenBank Accession no. (AN): DQ266104), 446 bp (AN: DQ266147), 518 bp (AN: DQ266165), 439 bp (AN: DQ266200), and 452 bp (AN: DQ266223) for genes Act, $\beta$-tub, Cal, H3, and ITS regions of rDNA, respectively. The amplification product of gene $\beta$-tub was polymorphic relative to that of $V$. albo-atrum isolates from lucerne and the amplicon of the ITS regions of rDNA was identical to that identified in nonlucerne $V$. albo-atrum isolates (46).

V. dahliae isolates amplified an identical sequence of about 200 $\mathrm{pb}$ for gene Act (AN: DQ266108-DQ266124) as well as an identical $\beta$-tub sequence of 448 bp (AN: DQ266129-DQ266138, DQ266140-DQ266145), except for isolates of VCG6. The latter amplified a sequence of 449 bp (AN: DQ266139) for gene $\beta-t u b$. Amplification of gene $\mathrm{Cal}$ from isolates of $\mathrm{V}$. dahliae yielded a product of 523 bp (ANs: DQ266154-DQ266163, DQ266166DQ266168, and DQ266174-DQ266177). All V. dahliae isolates but those of VCG2B ${ }^{334}$ amplified a fragment of 439 bp (ANs: DQ266180-DQ266182, DQ266184, DQ266185, and DQ266187- 
DQ266197) for gene $H 3$. VCG2B ${ }^{334}$ isolates amplified a sequence of 438 bp (ANs: DQ266183 and DQ266186) for this gene.

Amplification of the ITS regions of rDNA produced a DNA fragment of $452 \mathrm{bp}$ (ANs: DQ266204-DQ266216 and DQ266220-Q266222) from all $V$. dahliae isolates except those of VCG2A. These latter isolates yielded a 452-bp polymorphic sequence (AN: DQ266217-DQ266219) that was identical to that found in "short-spored" $V$. dahliae isolates from crucifers (16). Conversely, the ITS sequence from the remaining $V$. dahliae isolates showed $100 \%$ similarity with ITS sequences of other isolates of $V$. dahliae and some of $V$. dahliae var. longisporum (16).

Phylogenetic analysis of DNA sequences and the combined data set of AFLP fingerprints and DNA sequences. Phylogenetic trees for each DNA region derived from MP analysis (not shown) indicated that $V$. dahliae isolates were identical for most of the sequences, regardless of the VCG of isolates. Thus, individual gene genealogies provided very little resolution at the VCG level. The partition homogeneity test (PHT) for congruence among phylogenetic trees for sequences of Act, $\beta$-tub, Cal, H3, and ITS, and the $V$. dahliae-specific sequences indicated that trees were not significantly incongruent $(P=1)$. Therefore, data of all sequences were combined and subjected to MP analysis. First, the analysis was done using the Act, $\beta$-tub, $\mathrm{Cal}, \mathrm{H3}$, and the ITS sequences from $V$. dahliae and $V$. albo-atrum isolates. Results grouped isolates of VCG1A, VCG1B, VCG2B ${ }^{824}$, VCG4A, and VCG 4B; but placed isolates of VCG2B ${ }^{334}, \mathrm{VCG} 2 \mathrm{~A}$, and VCG6 each in separate clades (Fig. 2A). However, two main clades (lineages I and II) were identified when a similar analysis was done adding the $V$. dahliae-specific sequence to the above genes; lineage II being formed by two closely related subclades (Fig. 2B and C). Furthermore, subgroups differentiated within each clade correlated with VCGs. Lineage I included isolates of VCG1A, VCG1B, and VCG2 $\mathrm{B}^{334}$. Lineage II comprised isolates of VCG $2 \mathrm{~B}^{824}$ and VCG6 in one subclade and isolates of VCG4B and VCG2A in another. Location of VCG4A isolate 131M in those phylogenies varied among the four most parsimonious trees inferred by MP analysis, of which only two are shown in Figure $2 \mathrm{~B}$ and $\mathrm{C}$. In one case, VCG4A isolate 131M was placed close to subgroups comprising VCG2A and VCG4B isolates (Fig. 2B) but in another it was placed between lineage I (i.e., VCG1A, VCG1B, and VCG2 $\mathrm{B}^{334}$ ) and lineage II (i.e., VCG 2B ${ }^{824}$, VCG6, VCG4B, and VCG2A (Fig. 2C).

MP analysis of AFLP fingerprints from 18 Verticillium spp. isolates used for DNA sequencing (Table 1) led to inferring an MP phylogenetic tree (Fig. 3A). The tree topology was similar to that obtained from MP analysis of AFLP fingerprints of 90 Verticillium spp. isolates (Fig. 1). PHT for congruence of trees derived from analysis of DNA sequences and AFLP fingerprints showed no incongruence between them $(P=0.3)$ and allowed a subsequent phylogenetic analysis using the combined data sets of DNA sequences and AFLP fingerprints (Fig. 3B and C). The isolate of $V$. albo-atrum was excluded from that analysis because it did not amplify the $V$. dahliae-specific sequence. Results from analysis of the combined data of AFLP fingerprints and DNA sequences were similar to those obtained with AFLP data only and also concordant with results obtained with genealogies of all DNA sequences. Thus, the same two evolutionary lineages of $V$. dahliae VCGs could be inferred from analysis of the combined data sets, whereby: lineage I includes isolates of VCG1A, VCG1B, and VCG2 $\mathrm{B}^{334}$; and lineage II comprises isolates of VCG2B ${ }^{824}$, VCG4A, and VCG6 in one subclade, and isolates of VCG4B and VCG2A in another (Fig. 3B and C).

\section{DISCUSSION}

In a previous phenetic analysis of AFLP fingerprints from a large collection of $V$. dahliae isolates (14), we concluded that $V$. dahliae is a highly structured, clonal pathogen in which correla- tion between neutral genetic markers and vegetative compatibility can be used to infer relationships among VCGs. Such inference could not be done at that time because of the type of analysis performed with AFLP data. In the present work, phylogenetic analysis of AFLP fingerprints using 39 profiles from the previous study and 50 new profiles, as well as multiple gene genealogies not included in the earlier study, for the first time: (i) demonstrate evolutionary relationships among $V$. dahliae VCGs; (ii) identify two main evolutionary lineages that comprise different VCGs (i.e., lineage I: VCG1A, VCG1B, and VCG2B ${ }^{334}$; and lineage II: VCG2A VCG2B ${ }^{824}$, VCG4A, VCG4B, and VCG6); and (iii) show that VCGs have a monophyletic origin with the exception of VCG2B, that appears to be polyphyletic. Therefore, our results support Leslie's claim that assumptions of fungal isolates in a VCG sharing a common ancestor descent even if they are geographically isolated from one another should be taken with caution (38).

The VCG clonal lineages in $V$. dahliae and evolutionary relationships among them were resolved independently by analyses either of AFLP fingerprints, multiple gene genealogies, or the combined data set of AFLP fingerprints and multiple gene genealogies. AFLP markers are based on the simultaneous analysis of many loci representing the whole genome and bear high potential for phylogenetic studies $(20,25,45)$. However, these markers have not been widely used for phylogenetic inference presumably because they are considered highly homoplasic (18). In our study, the large number of AFLP markers analyzed and the moderate to high bootstrap values supporting the analysis indicate that AFLP fingerprints contained a sufficient phylogenetic signal to be used for the purpose of phylogenetic inferences at the intraspecific level in $V$. dahliae. In other studies, a qualitatively similar phylogenetic signal existed within AFLP fingerprint topologies generated by analyses of AFLP fingerprints and DNA sequences (45). Comparative use of different molecular markers in phylogenetic studies can provide resolution on different temporal scales. Thus, use of nucleotide variation of conserved sequences examines ancient patterns of population divergence (i.e., inter specific level) and AFLP fingerprints resolves population genetic structure on a more recent time scale (i.e., intra specific level). In our study, polymorphism level in five nuclear


Fig. 2. A, The most parsimonious tree inferred from maximum parsimony analysis using PAUP* of the combined set of sequences of genes actin, $\beta$ tubulin, calmodulin, and histone 3, and the ITS2 regions of rDNA from 17 Verticillium dahliae isolates representative of different vegetative compatibility groups (VCG). Tree length $(\mathrm{TL})=80$, consistency index $(\mathrm{CI})=1$, retention index $(\mathrm{RI})=1$, rescaled consistency index $(\mathrm{RC})=1$. V. albo-atrum was used to root the tree. $\mathbf{B}$ and $\mathbf{C}$, Two of the four most parsimonious trees inferred by maximum parsimony analysis using PAUP of the combined set sequences of the five loci in $\mathbf{A}$ and a $V$. dahliae-specific sequence (11) (TL = $25, \mathrm{CI}=0.88, \mathrm{RI}=0.963$, and $\mathrm{RC}=0.847)$. The $V$. dahliae-specific sequence from isolate V138 was used to root the trees. The trees were inferred using base substitutions and indels. The scale bar below each tree represents a single character change. Bootstrap values (1,000 replicates) are indicated in nodes. I, lineage I; II, and lineage II. 
DNA regions (i.e., actin, $\beta$-tubulin, calmodulin, and histone 3 genes, and the ITS 1 and 2 regions of the rDNA) among isolates of different VCGs was very low and provided little resolution at the VCG level individually; even combined, these makers provided little phylogenetic resolution (Fig. 2A). However, use of the $V$ dahliae-specific seq sequences amplified by primer pair DB19/ DB22 was much more productive than the other nuclear DNA markers and contributed to improved resolution (Fig. 2B and C). Of the four seq sequences identified in this work, seq1, seq2, and seq3 correlated with the VCG of isolates in agreement with an earlier report (15), indicating that this association is reproducible regardless of the variation in host source and geographic origin. The association of the newly reported sequence seq 7 with VCG4A would need confirmation using a larger set of diverse VCG4A isolates.

Bell (5) proposed that isolates of VCG1 and VCG4 may have evolved from VCG2 isolates. This proposition was based on the fact that VCG2 is distributed widely and VCG2 isolates occasionally establish weak complementation with those of VCG1 or VCG4, yet complementation between VCG1 and VCG4 isolates never occurs. Results of our study would support that hypothesis because each of the two evolutionary lineages identified comprises isolates of VCG2 (i.e., VGC2 $\mathrm{B}^{334}$ and $\mathrm{VCG} 2 \mathrm{~B}^{824}$ ). However, the evolutionary history of current $V$. dahliae VCGs had not been yet addressed. In filamentous fungi, such as $V$. dahliae, vegetative compatibility is governed by up to 10 het loci which most frequently determine allelic systems with two to four alleles per locus $(24,38,50)$. Incompatibility in allelic systems is triggered by co-expression of two different alleles at the same locus, whereas incompatibility in nonallelic systems results from the interaction of two genes belonging to different loci. Assuming asexual reproduction and clonality in $V$. dahliae, and that either genetic system governs vegetative compatibility of this fungus, two simple mechanisms are possible to evolve new VCGs. First,

TABLE 1. Verticillium isolates used in this study with vegetative compatibility grouping (VCG), previous reference, host source, geographic origin, and type of V. dahliae-specific sequence amplified

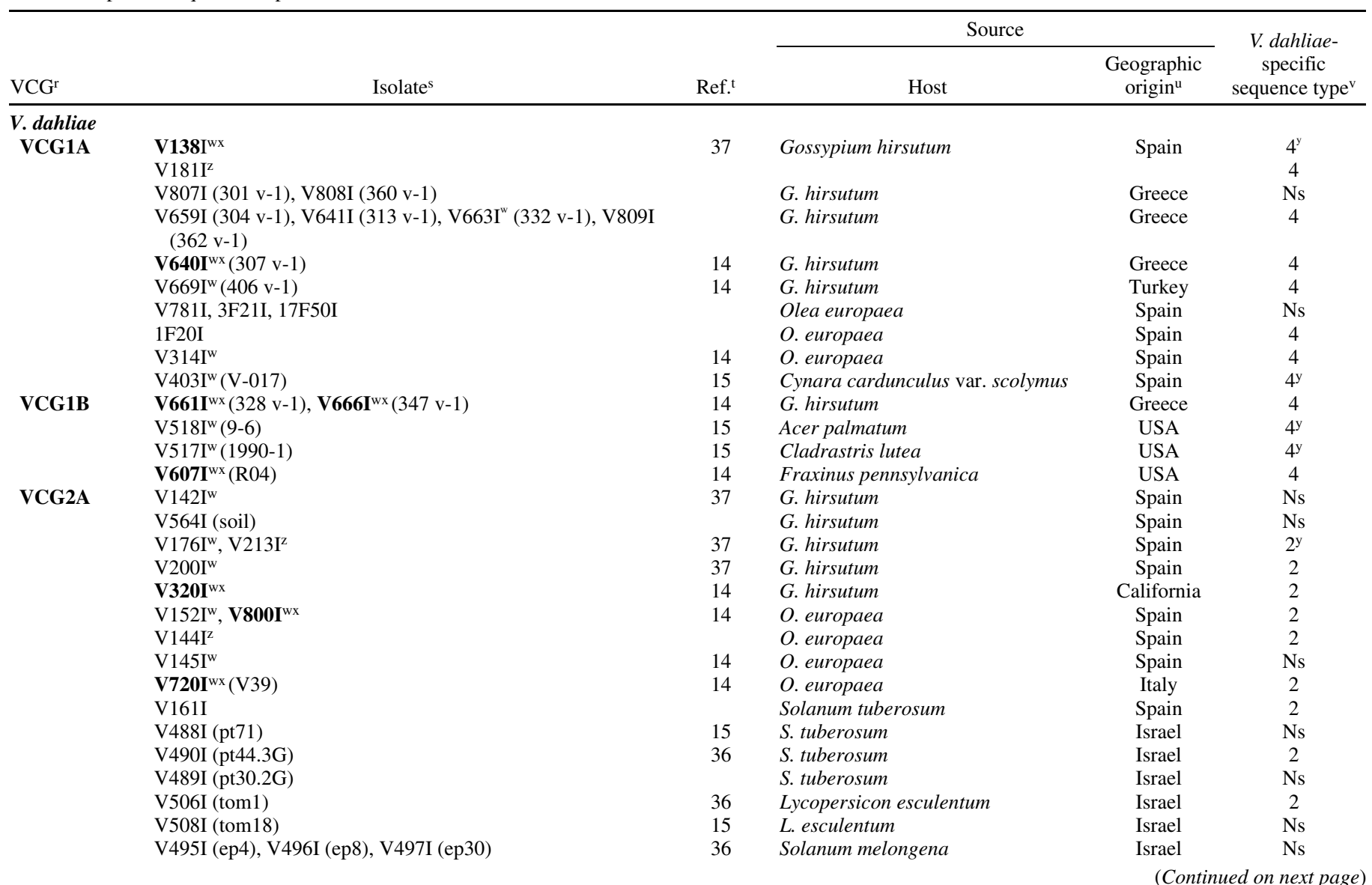

$\overline{\mathrm{r}}$ Vegetative compatibility was determined using nit mutants according to Korolev and Katan (35). VCG assessment in previous studies $(6,14,15,28,36,37)$ or carried out by other authors as unpublished results (ur) (R. Rowe; N. Korolev, J. Katan, R. M. Jiménez-Díaz, and T. Katan). HSI, heterokaryon self incompatible; $2 \mathrm{Ba}$, artichoke isolates able to complement with other artichoke isolates assigned to VCG2B but not with the international VCG testers; Nd, not determined.

s Isolate reference code according to IAS Collections. In brackets, code assigned to these isolates in previous studies or by the supplier.

${ }^{t}$ Reference of studies where these isolates were assigned to VCG. Isolates where no reference is given were assigned to VCG in this present study.

"Isolates from Greece provided by E. Paplomatas, Agricultural University of Athens, Athens, Greece; artichoke and almond isolates provided by J. Armengol, Universidad Politécnica de Valencia, Valencia, Spain; Isolates from Israel and VCG1B isolates from USA provided by T. Katan, The Volcani Center, Bet Dagan, Israel; Isolates from UK, California, and Brazil provided by D. Barbara, Warwick HRI, England; VCG4A isolates provided by R. Rowe, OARDC, The Ohio State University, Wooster, Ohio; isolates from Italy provided by F. Nigro, Università degli Studi di Bari, Bari, Italy, or A. Zazzerini, Università degli Studi di Perugia, Perugia, Italy.

${ }^{v}$ V. dahliae-specific sequence amplified in polymerase chain reaction assays using primer pair DB19/DB22 (11). 1, seq1 (527 pb); 2 , seq2 (526 pb); 3 , seq3 $(526 \mathrm{pb}) ; 4$, seq4 (542 pb); 5, seq5 (15); 7, seq7 (541 pb). Ns, not sequenced; -, there was no amplification.

${ }^{w}$ Isolates in which amplified fragment length polymorphism (AFLP) patterns were obtained by Collado-Romero et al. (14).

${ }^{x}$ Bold isolates used for sequencing and phylogenetic analysis of: actin, tubulin, calmodulin, and histone 3 genes; internal transcribed sequences of nuclear ribosomal RNA genes; and V. dahliae-specific DNA sequence (11).

y Sequenced by Collins et al. (15).

${ }^{\mathrm{z}}$ Isolates that were not used for AFLP analysis in this work. 
mutations might occur in a het locus that determine incompatibility with the parent isolate but allow formation of stable heterokaryons with its progeny. Alternatively, mutations might take place in genes that do not exist to limit heterokaryosis and thus cannot be considered het loci (50), but are representative of genes for which polymorphisms can bring about allelic variants whose co-expression determines deleterious effects. These two mechanisms would explain the appearance of monophyletic VCGs; i.e., once a new VCG becomes established others could be generated either directly from it or indirectly from those derived afterward once they become more or less differentiated.

Once several $V$. dahliae VCGs develop, new combinations of het loci could potentially develop as a result of parasexual recombination between isolates of different VCGs (26). The existence of 'bridging isolates' in $V$. dahliae, reportedly compatible with isolates from different VCGs $(4,33)$, would offer oppor- tunities for the development of heterokaryons and transient diploidy. However, our results cannot conclude whether parasexuality and generation of new het loci combinations have happened since in our study gene genealogies provided little resolution between VCGs.

VCG2B is a rather heterogeneous group of $V$. dahliae isolates in morphological and physiological traits as well as in host range and virulence $(28,33,36,37)$. Such heterogeneity could be associated with VCG2B polyphyly that suggests multiple independent origins of the set of het loci determining vegetative compatibility among isolates of that VCG. If changes in those loci were a common phenomenon, it might be expected that new $V$. dahliae VCGs would appear rather frequently. In reality, the VCG of a $V$. dahliae isolate is rather stable since, in general, an isolate does not change the ability to establish vegetative complementation with isolates of other VCGs $(33,47)$. Acquisition of a common set

\begin{tabular}{|c|c|c|c|c|c|}
\hline \multirow[b]{2}{*}{$\mathrm{VCG}^{\mathrm{r}}$} & \multirow[b]{2}{*}{ Isolate $^{s}$} & \multirow[b]{2}{*}{ Ref. $^{t}$} & \multicolumn{2}{|l|}{ Source } & \multirow{2}{*}{$\begin{array}{c}\text { V. dahliae- } \\
\text { specific } \\
\text { sequence type }^{\mathrm{V}}\end{array}$} \\
\hline & & & Host & $\begin{array}{c}\text { Geographic } \\
\text { origin }^{\mathrm{u}}\end{array}$ & \\
\hline \multirow[t]{7}{*}{ VCG2A } & V498I (ep53) & 15 & S. melongena & Israel & Ns \\
\hline & $\mathrm{V} 404 \mathrm{I}^{\mathrm{z}}(\mathrm{V}-018)$ & 28 & C. cardunculus var. scolymus & Spain & $2^{z}$ \\
\hline & V398I & & Cucurbita pepo & Spain & Ns \\
\hline & V394I & & Cucumis melo & Spain & Ns \\
\hline & V121I & & Citrullus lanatus & Spain & Ns \\
\hline & V556I (128) & & Brassica oleracea var. italica & Germany & 2 \\
\hline & V562I (JB84015a) & & Unknown & Japan & Ns \\
\hline \multirow[t]{18}{*}{ VCG2B } & $\mathbf{V 3 5 7 I ^ { \mathrm { wx } } ( J Y )}$ & 14 & G. hirsutum & China & 1 \\
\hline & V649I ${ }^{\mathrm{w}}(361 \mathrm{v}-1)$ & 14 & G. hirsutum & Greece & Ns \\
\hline & $\mathrm{V} 282 \mathrm{I}^{\mathrm{w}}(\cot 67)$ & 36 & G. hirsutum & Israel & 1 \\
\hline & V302I (cot117) & & & & $1^{y}$ \\
\hline & V510I' ${ }^{\mathrm{w}}($ tom 20$)$ & 36 & L. esculentum & Israel & 1 \\
\hline & V511I (tom28) & 15 & L. esculentum & Israel & Ns \\
\hline & V499I (ep1) & 15 & S. melongena & Israel & Ns \\
\hline & V500I (ep22) & & & & 1 \\
\hline & V594I ${ }^{\mathrm{w}}, \mathrm{V}^{2} 13 \mathrm{I}^{\mathrm{wx}}, \mathrm{V} 700 \mathrm{I}^{\mathrm{z}}$ & 28 & C. cardunculus var. scolymus & Spain & 4 \\
\hline & V396I ${ }^{\mathrm{wx}}(\mathrm{V}-008)$ & 15 & C. cardunculus var. scolymus & Spain & $1^{\mathrm{y}}$ \\
\hline & V479I ${ }^{\mathrm{w}}, \mathrm{V} 593 \mathrm{I}^{\mathrm{w}}$ & 28 & C. cardunculus var. scolymus & Spain & 1 \\
\hline & V552I (332) & & Fragaria ananassa & England & $1^{y}$ \\
\hline & V554I (MD71) & 15 & Matricaria chamomilla & Germany & $1^{\mathrm{y}}$ \\
\hline & V555I (MD124) & & & & 1 \\
\hline & V390Iz $(V-001)$ & 15 & Prunus amigdalus & Spain & $4^{y}$ \\
\hline & $\mathrm{V} 408 \mathrm{I}^{\mathrm{Z}}(\mathrm{V}-022)$ & 15 & P. amigdalus & Spain & $1^{\mathrm{y}}$ \\
\hline & V258I (CECE) & & Cicer arietinum & Italy & Ns \\
\hline & V563I (JC84160a) & & Unknown & Japan & 1 \\
\hline $2 \mathrm{Ba}$ & V702I ${ }^{\mathrm{wx}}$ & 28 & C. cardunculus var. scolymus & Spain & 4 \\
\hline VCG4A & 131-M $\mathbf{M}^{\mathrm{x}}, 171-4 \mathrm{~A}^{\mathrm{z}}$ & 31 , ur & S. tuberosum & USA & 7 \\
\hline \multirow[t]{16}{*}{ VCG4B } & V192I ${ }^{\mathrm{w}}$ & 37 & G. hirsutum & Spain & Ns \\
\hline & $\mathrm{V} 160 \mathrm{I}^{\mathrm{w}}(\cot 24), \mathrm{V} 275 \mathrm{I}(\cot 23)$ & 36 & G. hirsutum & Israel & Ns \\
\hline & V279I ${ }^{\mathrm{w}}(\cot 40), \mathrm{V} 294 \mathrm{I}^{\mathrm{z}}(\cot 92), \mathbf{V 3 0 4} \mathrm{I}^{\mathrm{wx}}(\cot 120)$ & 37 & G. hirsutum & Israel & 2 \\
\hline & V673I (431 v-1) & & O. europaea & Greece & Ns \\
\hline & V392I ${ }^{\mathrm{w}}, \mathrm{V} 803 \mathrm{I}^{\mathrm{w}}$ & 14 & O. europaea & Spain & 2 \\
\hline & V491I (pt15) & 36 & S. tuberosum & Israel & Ns \\
\hline & V492I (pt63) & 15 & S. tuberosum & Israel & 2 \\
\hline & V494I (pt103.1G) & & S. tuberosum & Israel & 2 \\
\hline & V515I (tom5) & 36 & L. esculentum & Israel & 2 \\
\hline & V513I (tom26) & 37 & L. esculentum & Israel & Ns \\
\hline & V557I (P14) & & L. esculentum & Brazil & $2^{y}$ \\
\hline & V502I (ep17) & 37 & S. melongena & Israel & 2 \\
\hline & V505I (ep47) & & & & Ns \\
\hline & V503I (ep24) & & S. melongena & Israel & Ns \\
\hline & V684I ${ }^{\mathrm{wx}}$ & 28 & C. cardunculus var. scolymus & Spain & 2 \\
\hline & V551I (330), V553I (1875) & & $F$. ananassa & England & Ns \\
\hline \multirow[t]{2}{*}{ VCG6 } & V560I ${ }^{\mathrm{wx}}(\mathrm{VdCa} 83 \mathrm{a})$ & 6 & Capsicum annuит & California & 1 \\
\hline & V561I ${ }^{\mathrm{w}}(\mathrm{VdCa} 147 \mathrm{a})$ & & & & $1^{\mathrm{y}}$ \\
\hline \multirow[t]{3}{*}{ HSI } & $\mathrm{V} 4 \mathrm{I}^{\mathrm{w}}$ & 37 & G. hirsutum & Spain & 2 \\
\hline & V537I ${ }^{\mathrm{z}}, \mathrm{V} 547 \mathrm{I}^{\mathrm{w}}$ & 28 & C. cardunculus var. scolymus & Spain & 4 \\
\hline & V658I ${ }^{\mathrm{w}}(398 \mathrm{v}-1)$ & 14 & O. europaea & Cyprus & Ns \\
\hline $\mathrm{Nd}$ & V810I (340 v-1) & & G. hirsutum & Greece & Ns \\
\hline $\mathrm{Nd}$ & V448I (Vd-307) & & L. esculentum & Italy & 1 \\
\hline \multicolumn{6}{|c|}{ V. albo-atrum } \\
\hline $\mathrm{Nd}$ & $\mathbf{V 0 4 8 I}^{\mathrm{wx}}(\mathrm{V}-48 \mathrm{I})$ & & Humulus lupulus & England & - \\
\hline
\end{tabular}


of het loci that determines vegetative compatibility between two isolates could occur by means of independent mutation events. Alternatively, an isolate might have a regressive change to recover ability of complementing with isolates of an ancestral VCG with which it was previously compatible. In that scenario, our results would suggest that VCG2 $\mathrm{B}^{334}$ isolates probably derived from VCG1A isolates (or from a recent common ancestor) because, as a group, VCG2 $\mathrm{B}^{334}$ is molecularly more similar to VCG1A and VCG1B than to VCG2B ${ }^{824}$. Also, VCG2 ${ }^{334}$ isolates have probably emerged in eastern-central Spain because they have not been identified yet in any other place. Polyphyly in some $V$. dahliae VCG subgroups may be important for the control of Verticillium wilt diseases. That some isolates in polyphyletic VCG subgroups have evolved more than once in different places implies that their ecological, physiological, and/or virulence traits might vary across locations as compared with those of original populations.

VCG $2 \mathrm{~B}^{334}$ is also rather genetically heterogeneous because it comprises a group of isolates (namely $\mathrm{VCG} 2 \mathrm{Br}^{334}$ ) that are compatible with the international reference VCG2B testers, and another group of isolates (namely VCG2Ba) that did not complement with the reference testers but complemented with VCG $2 \mathrm{Br}^{334}$ isolates. However, VCG2Ba and VCG2Br ${ }^{334}$ subgroups could not be molecularly differentiated by ColladoRomero et al. (14); therefore, the loss (or gain) of ability of their isolates to establish vegetative complementation with isolates of the corresponding VCG2B subgroup must be a recent event. The evolutionary history of VCG2 $\mathrm{B}^{824}$ isolates differed from that of VCG2 $\mathrm{B}^{334}$. VCG2 $\mathrm{B}^{824}$ might have originated from the common VCG ancestor and evolved in lineage II together with the recently described VCG6. Isolates of VCG6 probably originated from VCG2 $\mathrm{B}^{824}$ as suggested by molecular similarities and analysis of gene genealogies (Fig. 2B and C). This might have happened in the Salinas Valley (California) where VCG6 recently was identified as a new VCG and both VCG2B and VCG4B have been reported to occur extensively (6). Alternatively, VCG6 might have originated together with VCG4A as suggested by the combined analysis of AFLP markers and DNA sequences (Fig. 3).

VCG1A, VCG1B, and VCG2B ${ }^{334}$ comprised in lineage I were the least related to other VCGs and thus must have diverged prior to differentiation of lineage II. Differentiation between VCG1A and VCG1B was possible only by analysis of AFLP fingerprints which would suggest a recent event of differentiation between them. Furthermore, VCG1B isolates originating from the United States were molecularly differentiated from those originating from Greece; these two groups of isolates were separated in the trees derived from MP analysis of combined AFLP and DNA sequence data (Fig. 3B and C) as well as individual AFLP data (Fig. 1). This would suggest that VCG1B might have originated from VCG1A isolates in North America and Greece independently and thus could have a polyphyletic origin. However, further studies of a larger number of VCG1B isolates representatives from the two locations are needed to confirm that hypothesis.

Lineage II comprised two closely related groups of VCGs, whereby V. dahliae isolates of VCG2B ${ }^{824}$ and VCG6 were clearly separated from those of VCG2A and VCG4B. $V$. dahliae isolates of VCG4B and VCG2A appear to be closely related but they were not differentiated into two clear subgroups in the analysis of combined data (Fig. 3B and C). These two VCGs were placed in two different subgroups of lineage II by MP analysis of gene genealogies (Fig. 2B and C), but such a differentiation was less clear from AFLP analysis of a larger number of isolates which suggested that VCG2A isolates formed three molecular groups (Fig. 1). These molecular differences could be due to recent evolutionary events and probably are associated with the geographic localization and/or host selection of isolates or homoplasy of AFLP data. This also concerns location of VCG4B isolates from artichoke, that were closer to VCG2A than to other VCG4B isolates when phylogenetic analysis of a large number of isolates was carried out with AFLP data only (Fig. 1), whereas use of gene genealogies supports monophyly of VCG4B

A


10

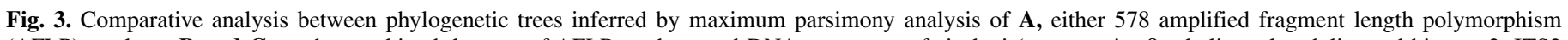



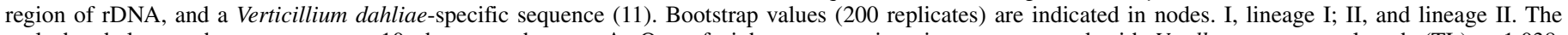

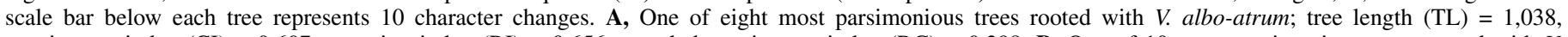

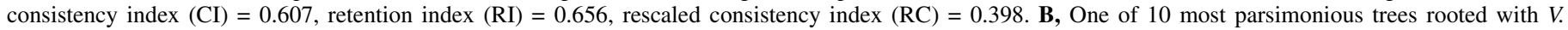


the phylogenies estimated from AFLP and combined analysis of AFLP and DNA sequence data. C, The same tree as in B, but unrooted. 
(including artichoke isolate) (Fig. 2B and C). The use of AFLP marker alone could mask phylogenetic relationships among $V$. dahliae VCGs because of homoplasy and comparisons of phylogenies obtained by the two approaches may reveal important relationships.

The phylogenetic relationships of the single VCG4A isolate $131 \mathrm{M}$ in the study with other VCGs is uncertain. Thus far, VCG4A has been found only in North America in association with potato crops (48). The VCG4A isolate was placed between lineages I and II or assigned to same subgroup of VCG2A and VCG4B in lineage II by MP analysis of gene genealogies, and assigned to a subgroup with VCG2 ${ }^{824}$ and VCG6 in lineage II by MP analysis of AFLP fingerprints (Figs. $2 \mathrm{~B}$ and $\mathrm{C}$ and $3 \mathrm{~A}$ ). Such inconsistency would be in line with isolates of VCG4A sharing some molecular features that differentiate among other VCGs. While isolates of other V. dahliae VCGs yielded either the 334- or 824-bp markers in PCR assays using specific primers $(14,28)$, use of these same primers for assays of VCG4A isolates yielded those two amplicons (Collado-Romero, Mercado-Blanco, and JiménezDíaz, unpublished data). Nevertheless, data from our work do not rule out the possibility of VCG4A isolates were derived from hybridization events between isolates of VCG1A and those in subgroups of lineage II (VCG2 ${ }^{824}$ and VCG6, or VCG2A and VCG4B).

In summary, our study demonstrates that a phylogenetic relationship exists between groups of $V$. dahliae VCGs, with most of them being monophyletic and VCG2B being polyphyletic. Moreover, if our study had been limited to results derived from analysis of AFLP fingerprints some interesting conclusions would not have been revealed (e.g., the origin of VCG2B ${ }^{334}$ isolates). Similarly, limiting our study to analysis of DNA sequences would have obscured intra-VCG differentiation (e.g., the fingerprint data resolve genotypes within recently evolving clonal lineages as VCG1 and VCG2A that were not resolved by analysis of sequence data of six loci). Therefore, analysis of AFLP fingerprints or DNA sequences or a combination of the two was a highly valuable method for the identification of phylogenetic relationships at the intraspecific level in $V$. dahliae.

\section{ACKNOWLEDGMENTS}

This research was supported by grants AGL2000-1444 and AGL200300503 from Comisión Interministerial de Ciencia y Tecnología (CICYT) of Spain, and 'Verticilosis del olivo' from Fundación Ramón Areces. M. Collado-Romero was a recipient of an FPI fellowship from the Spanish Ministry of Education and Science (MEC) and J. Mercado-Blanco was a contract holder under the "Ramón y Cajal" Programme of MEC. We thank M. M. Jiménez-Gasco for helpful comments and suggestions on the manuscript prior to submission, J. Armengol and J. García-Jiménez for providing $V$. dahliae isolates from artichoke, R. C. Rowe and T. Katan for providing reference strains of $V$. dahliae VCGs and Israeli nit testers, respectively, and $\mathrm{H}$. Mueller who provided primers Tavo1 and 2. Editorial improvement from anonymous reviewers and senior editor is gratefully acknowledged.

\section{LITERATURE CITED}

1. Apple, D., and Gordon, T. R. 1995. Intraspecific variation within populations of Fusarium oxysporum based on RFLP analysis of the intergenic spacer region of the rDNA. Exp. Mycol. 19:120-128.

2. Apple, D., and Gordon, T. R. 1996. Relationships among pathogenic and nonpathogenic isolates of Fusarium oxysporum based on the partial sequence of the intergenic spacer region of the ribosomal DNA. Mol. Plant-Microbe Interact. 9:125-138.

3. Baayen, R. P., O'Donnell, K., Bonants, P. J. M., Cilgenik, E., Kroon, L. P. N. M., Roebroeck, E. J. A., and Waalwijk, C. 2000. Gene genealogies and AFLP analyses in the Fusarium oxysporum complex identify monophyletic and nonmonophyletic formae speciales causing wilt and root rot diseases. Phytopathology 90:891-900.

4. Barbara, D. J., and Clewes, E. 2003. Plant pathogenic Verticillium species: How many of them are there. Mol. Plant Pathol. 4:297-305.
5. Bell, A. A. 1994. Mechanisms of disease resistance in Gossypium species and variation in Verticillium dahliae. Pages 225-235 in: Proc. World Cotton Res. Conf.1. G. A. Constable and N. W Forrester, eds. CSIRO, Melbourne.

6. Bhat, R. G., Smith, R. F., Koike, S. T., Wu, B. M., and Subbarao, K. V. 2003. Characterization of Verticillium dahliae isolates and wilt epidemics of pepper. Plant Dis. 87:789-797.

7. Bejarano-Alcázar, J., Blanco-López, M. A., Melero-Vara, J. M., and Jiménez-Díaz, R. M. 1996. Etiology, importance, and distribution of Verticillium wilt of cotton in southern Spain. Plant Dis. 80:1233-1238.

8. Bidochka, M. L., Leger, R. J. S., Stuart, A., and Gowanlock, K. 1999. Nuclear rDNA phylogeny in the fungal genus Verticillium and its relationship to insect and plant virulence, extracellular proteases and carbohydratases. Microbiology 145:955-963.

9. Carbone, I., Anderson, J. B., and Kohn, L. M. 1999. Patterns of descent in clonal lineages and their multilocus fingerprints are resolved with combined gene genealogies. Evolution 53:11-21.

10. Carbone, I., and Kohn, L. M. 1999. A method for designing primer sets for speciation studies in filamentous ascomycetes. Mycologia 91:553-556.

11. Carder, J. H., Morton, A., Tabrett, A. M., and Barbara, D. J. 1994. Detection and differentiation by PCR of subspecific groups within two Verticillium species causing vascular wilts in herbaceous hosts. Pages 9197 in: Modern Assays for Plant Pathogenic Fungi. A. Schots, F. M. Dewey, and R. Oliver, eds. CAB International, Oxford.

12. Coleman, A. W. 2003. ITS2 is a double-edged tool for eukaryote evolutionary comparisons. Trends Genet. 19:370-375.

13. Chen, W. 1994. Vegetative compatibility groups of Verticillium dahliae from ornamental woody plants. Phytopathology 84:214-219.

14. Collado-Romero, M., Mercado-Blanco, J., Olivares-García, C., ValverdeCorredor, A., and Jiménez-Díaz, R. M. 2006. Molecular variability within and among Verticillium dahliae vegetative compatibility groups determined by fluorescent amplified fragment length polymorphism and polymerase chain reaction markers. Phytopathology 96:485-495.

15. Collins, A., Mercado-Blanco, J., Jiménez-Díaz, R. M., Olivares, C., Clewes, E., and Barbara, D. J. 2005. Correlation of molecular markers and biological properties in Verticillium dahliae and the possible origins of some isolates. Plant Pathol. 54:549-557.

16. Collins, A., Okoli, C. A. N., Morton, A., Parry, D., Edwards, S. G., and Barbara, D. J. 2003. Isolates of Verticillium dahliae pathogenic to crucifers are of at least three distinct molecular types. Phytopathology 93:364-376.

17. Correll, J. C., Klittich, C. J. R., and Leslie, J. F. 1987. Nitrate nonutilizing mutants of Fusarium oxysporum and their use in vegetative compatibility tests. Phytopathology 77:1640-1646.

18. Deprés, L., Gielly, L., Redoutet, B., and Tabarlet, P. 2003. Using AFLP to resolve phylogenetic relationships in a morphologically diversified plant species complex when nuclear and chloroplast sequences fail to reveal variability. Mol. Phylogenet. Evol. 27:185-196.

19. Fahleson, J., Hu, Q., and Dixelius, C. 2004. Phylogenetic analysis of Verticillium species based on nuclear and mitochondrial sequences. Arch. Microbiol. 181:435-442.

20. Fahleson, J., Lagercrantz, U., Hu, Q., Stevenson, L. A., and Dixelius, C. 2003. Estimation of genetic variation among Verticillium isolates using AFLP analysis. Eur. J. Plant Pathol. 109:361-371.

21. Farris, J. S., Källersjö, A. G., Kluge, A. G., and Bult, C. 1995. Testing significance of incongruence. Cladistic 10:315-319.

22. Garzón, C. D., Geiser, D. M., and Moorman, G. W. 2005. Amplified fragment length polymorphism analysis and internal transcribed spacer and coxII sequences reveal a species boundary within Pythium irregulare. Phytopathology 95:1489-1498.

23. Glass, N. L., and Donaldson, G. C. 1995. Development of primer sets designed for use with the PCR to amplify conserved genes from filamentous ascomycetes. Appl. Environ. Microbiol. 61:1323-1330.

24. Glass, N. L., and Kaneko, I. 2003. Fatal attraction: Nonself recognition and heterokaryon incompatibility in filamentous fungi. Eukaryot. Cell 2:1-8.

25. Groenewald, S., Van den Berg, N., Marasas, W. F. O., and Vijloen, A. 2006. The application of high-throughput AFLP's in assessing genetic diversity in Fusarium oxysporum f. sp. cubense. Mycol. Res. 110:297305 .

26. Hastie, A. C. 1981. The genetics of conidial fungi. Pages 511-543 in: Biology of Conidial Fungi Vol. 2. G. T Cole and W. B Kendrick, eds. Academic Press, New York.

27. Huelsenbeck, J. P., Bull, J. J., and Cunningham, C. W. 1996. Combining data in phylogenetic analysis. Trends Ecol. Evol. 11:152-158.

28. Jiménez-Díaz, R. M., Mercado-Blanco, J., Olivares-García, C., ColladoRomero, M., Bejarano-Alcázar, J., Rodríguez-Jurado, D., Giménez-Jaime, A., García-Jiménez, J., and Armengol, J. 2006. Genetic and virulence diversity in Verticillium dahliae populations infecting artichoke in eastern-central Spain. Phytopathology 96:288-298. 
29. Jiménez-Gasco, M. M., Milgroom, M. G., and Jiménez-Díaz, R. M. 2002. Gene genealogies support Fusarium oxysporum f. sp. ciceris as a monophyletic group. Plant Pathol. 51:72-77.

30. Joaquim, T. R., and Rowe, R. C. 1990. Reassessment of vegetative compatibility relationships among strains of Verticillium dahliae using nitrate-nonutilizing mutants. Phytopathology 80:1160-1166.

31. Joaquim, T. R., and Rowe, R. C. 1991. Vegetative compatibility and virulence strains of Verticillium dahliae from soil and potato plant. Phytopathology 81:552-558.

32. Jukes, T. H., and Cantor, C. R. 1969. Evolution of protein molecules. Pages 21-132 in: Mammalian Protein Metabolism. H. H. Munro, ed. Academic Press, New York.

33. Katan, T. 2000. Vegetative compatibility in populations of VerticilliumAn overview. Pages 69-86 in: Advances in Verticillium: Research and Disease Management. E. C. Tjamos, R. C. Rowe, J. B. Heale, and R. D. Fravel, eds. American Phytopathological Society Press, St. Paul, MN.

34. Koike, S. T., Subbarao, K. V., Davis, R. M., Gordon, T. R., and Hubbard, J. C. 1994. Verticillium wilt of cauliflower in California. Plant Dis. 78:1116-1121.

35. Korolev, N., and Katan, T. 1997. Improved medium for selecting nitrate nonutilizing (nit) mutants of Verticillium dahliae. Phytopathology 87:1067-1070.

36. Korolev, N., Katan, J., and Katan, T. 2000. Vegetative compatibility groups of Verticillium dahliae in Israel: Their distribution and association with pathogenicity. Phytopathology 90:529-566.

37. Korolev, N., Pérez-Artés, E., Bejarano-Alcázar, J., Rodríguez-Jurado, D., Katan, J., Katan, T., and Jiménez-Díaz, R. M. 2001. Comparative study of genetic diversity and pathogenicity among populations of Verticillium dahliae from cotton in Spain and Israel. Eur. J. Plant Pathol. 107:443-456.

38. Leslie, J. F. 1993. Fungal vegetative compatibility. Annu. Rev. Phytopathol. 31:127-150.

39. McDonald, D. A., and Linde, C. 2002. Pathogen population genetics, evolutionary potential, and durable resistance. Annu. Rev. Phytopathol. 40:349-379.

40. Mercado-Blanco, J., Rodríguez-Jurado, D., Parrilla-Araujo, S., and Jiménez-Díaz, R. M. 2003. Simultaneous detection of the defoliating and nondefoliating Verticillium dahliae pathotypes in infected olive plants by duplex, nested polymerase chain reaction. Plant Dis. 87:1487-1494.

41. Mercado-Blanco, J., Rodríguez-Jurado, D., Pérez-Artés, E., and JiménezDíaz, R. M. 2001. Detection of the nondefoliating pathotype of Verticillium dahliae in infected olive plants by nested PCR. Plant Pathol. 50:609-619.

42. O’Donnell, K., Kistler, H. C., Cigelnik, E., and Ploetz, R. C. 1998. Multiple evolutionary origins of the fungus causing Panama disease of banana: Concordant evidence from nuclear and mitochondrial gene genealogies. Proc. Natl. Acad. Sci. USA 95:2044-2049.

43. Pantou, M. P., Strunnikova, O. K., Shakhnazarova, V. Y., Vishnevskaya, N. A., Papalouka, V. G., and Typas, M. A. 2005. Molecular and immunochemical phylogeny of Verticillium species. Mycol. Res. 109:889-902.

44. Pegg, G. F., and Brady, B. L. 2002. Verticillium Wilts. CAB International, Oxford.

45. Pelser, P. B., Gravendeel, B., and van der Meijden, R. 2003. Phylogeny reconstruction in the gap between too little and too much divergence: the closest relatives of Senecio jacobaea (Asteraceae) according to DNA sequences and AFLPs. Mol. Phylogenet. Evol. 29:613-628.

46. Qin, Q.-M., Vallad, G. E., Wu, B. M., and Subbarao, K. V. 2006. Phylogenetic analyses of phytopathogenic isolates of Verticillium spp. Phytopathology 96:582-592.

47. Rowe, R. C. 1995. Recent progress in understanding relationships between Verticillium species and subspecific groups. Phytoparasitica 23:31-38.

48. Rowe, R. C., and Powelson, M. L. 2002. Potato early dying: Management challenges in a changing production environment. Plant Dis. 86:11841193.

49. Saitou, N., and Nei, M. 1987. The neighbour-joining method: A new method for reconstructing phylogenetic trees. Mol. Biol. Evol. 4:406-425.

50. Saupe, S. J., Clavé, C., and Bégueret, J. 2000. Vegetative incompatibility in filamentous fungi: Podospora and Neurospora provide some clues. Curr. Opin. Microbiol. 3:608-612.

51. Strausbaugh, C. A. 1993. Assessment of vegetative compatibility and virulence of Verticillium dahliae isolates from Idaho potatoes and tester strains. Phytopathology 83:1253-125.

52. Strausbaugh, C. A., Schroth, M. N., Weinhold, A. R., and Hancock, J. G. 1992. Assessment of vegetative compatibility of Verticillium dahliae tester strains and isolates from California potatoes. Phytopathology 82:6168 .

53. Subbarao, K. V., Hubbard, J. C., Greathead, A. S., and Spencer, G. A. 1997. Verticillium wilt. Pages 26-27 in: Compendium of Lettuce Diseases. R. M. Davis, K. V. Subbarao, R. N. Raid, and E. A. Kurzt, eds. American Phytopathological Society Press, St. Paul, MN.

54. Talboys, P. W. 1960. A culture medium aiding the identification of Verticillium albo-atrum and V. dahliae. Plant Pathol. 9:58-59.

55. Van de Peer, Y. 2003. Analysis of nucleotide sequences using TREECON. Pages 101-136 in: The Phylogenetic HandBook: A Practical Approach to DNA and Protein Phylogeny. M. Salemi and A. M Vandamme, eds. Cambridge University Press, Cambridge.

56. Volossiouk, T., Robb, E. J., and Nazar, R. N. 1995. Direct DNA extraction for PCR-mediated assays of soil organisms. Appl. Environ. Microbiol. 61:3972-3976 